\title{
The effect of dobutamine in sepsis: a propensity score matched analysis
}

\author{
Youfeng Zhu ${ }^{1 \dagger}$, Haiyan Yin ${ }^{2 \dagger}$, Rui Zhang ${ }^{1}$, Xiaoling Ye ${ }^{1}$ and Jianrui We ${ }^{3^{*}}$
}

\begin{abstract}
Background: The use of dobutamine in patients with sepsis is questionable currently. As the benefit of dobutamine in septic patients is unclear, we aimed to evaluate whether the use of dobutamine was associated with decreased hospital mortality in sepsis patients.

Methods: Based on the analysis of MIMIC III public database, we performed a big-data, real world study. According to the use of dobutamine or not, patients were categorized as the dobutamine group or non dobutamine group. We used propensity score matched (PSM) analysis to adjust for confoundings. The primary outcome was hospital mortality.
\end{abstract}

Results: In the present study, after screening 38,605 patients, 2826 patients with sepsis were included. 121 patients were in dobutamine group and 2165 patients were in non dobutamine group. Compared with patients in non-dobutamine group, patients in dobutamine group had a lower MAP, higher HR, higher RR, higher severity of illness scores. 72 of 121 patients (59.5\%) in the dobutamine group and 754 of 2165 patients (34.8\%) in the non-dobutamine group died in the hospital, which resulted in a significant between-group difference (OR 1.56, 95\% Cl 1.01-2.40; $\mathrm{P}=0.000)$. For the secondary outcomes, patients in dobutamine group received more MV use, more renal replacement therapy use, had longer ICU stay durations and more cardiac arrhythmias than those in non-dobutamine group. After adjusting for confoundings between groups by PSM analysis, hospital mortality was consistently higher in dobutamine group than that in non-dobutamine group (60.2\% vs. $49.4 \%$, OR $1.55,95 \% \mathrm{Cl} 1.01-2.37 ; \mathrm{P}=0.044)$.

Conclusions: Among patients with sepsis, our study showed that the use of dobutamine was not associated with decreased hospital mortality. Further large scale, randomized controlled studies are warrented to confirm our findings.

Keywords: Dobutamine, Sepsis, Propensity score matched analysis, Hospital mortality

\section{Background}

Dobutamine is recommended for septic patients who have myocardial depression according to surviving sepsis campaign guidelines, especially in those with evidence of persistent hypoperfusion, despite sufficient fluid resuscitation and the administration of vasopressor drugs [1]. However, there is uncertainty whether it has robust

\footnotetext{
*Correspondence: Jianruiw@163.com

†Youfeng Zhu and Haiyan Yin are co-first authors

${ }^{3}$ Guangzhou Women and Children's Medical Center, No. 9 Jinsui Road,

Guangzhou 510220, Guangdong, China

Full list of author information is available at the end of the article
}

effects on patient centered outcomes such as mortality. The data supporting the use of dobutamine are mainly physiologic, which may improve some variables of perfusion and ameliorate hemodynamics, such as improving central venous oxygen saturation and decreasing lactate levels [1].

So far, no randomized controlled trials have performed to evaluate whether there are significant differences between dobutamine and placebo on clinical outcomes in septic patients. In the three EGDT validation studies (ProMISe, ProCESS and ARISE studies), the use of dobutamine was more frequent in the EGDT group than in the control group (ProMISe study 8.0 vs. $1.1 \%$, respectively, 
$\mathrm{p}<0.001$; ProCESS study 8.0 vs. $1.1 \%$, respectively, $\mathrm{p}<0.001$; and ARISE study 15.4 vs. $2.6 \%$, respectively, $\mathrm{p}<0.0001$ ), but the mortality outcomes were similar in both group [2-4]. And the adverse effects with the use of dobutamine were not detected in the previous studies.

Dobutamine increases myocardial oxygen consumption, raises myocardial work, reduces cardiac efficiency, although it can increase cardiac index $[5,6]$. Furthermore, some studies reported the use of dobutamine was cardiotoxic, which might induce eosinophilic cardiomyopathy or takotsubo cardiomyopathy [7-9]. Hence, the use of dobutamine in patients with sepsis is questionable currently. As the benefit of dobutamine in septic patients is unclear so far, in this study, we aimed to evaluate whether the use of dobutamine was associated with decreased hospital mortality in sepsis patients.

\section{Methods}

We performed this study in accordance with the STrengthening the Reporting of OBservational studies in Epidemiology (STROBE) statement [10]. This was a bigdata, real world study based on the third edition of the Medical Information Mart in Critical Care (MIMICIII) database, which was developed and maintained by the Laboratory for Computational Physiology at MIT [11, 12] The MIMICIII database included longitudinal data on 38,605 patients who were admitted to the ICU of Beth Israel Deaconess Medical Center from 2002 to 2011 for a total of 53,423 distinct admissions. As the present study was based on the analysis of the MIMICIII public database, ethical review and informed consent were waived.

\section{Patients}

We screened the discharge diagnosis of patients in MIMIC III database by ICD 9 and ICD 10 codes. Adult patients (age $\geq 18$ years) who had a discharge diagnosis of sepsis, severe sepsis or septic shock and were admitted to the ICU from 2002 to 2011 in the MIMICIII database were screened for inclusion.

Patients who were under age 18, were pregnant, had obstructive hypertrophic cardiomyopathy, or had no detailed demographic information, were excluded.

The patients who used dobutamine during ICU stay were categorized as the dobutamine group, with the remaining patients were categorized as the non dobutamine group.

\section{Sepsis diagnosis}

The definitions and diagnositic criteria for sepsis, severe sepsis and septic shock were unchanged between 2002 and 2011, according to the Surviving Sepsis Campaign Guidelines [1, 13, 14].

\section{Data collection}

In this study, only each patient's first ICU admission data were included. The following demographic data and admission information were collected: age, gender, weight, height, body mass index (BMI), mean arterial pressure (MAP), heart rate (HR), respiratory rate (RR), temperature, Simplified Acute Physiology Score-I (SAPSI), Simplified Acute Physiology Score-II (SAPS-II), Sequential Organ Failure Assessment (SOFA), Elixhauser comorbidity index (ECI), admission type (emergency or elective), sepsis type (sepsis, severe sepsis, septic shock).

Additionally, data regarding the use of mechanical ventilation (MV) or renal replacement therapy (RRT) within the first day of ICU admission were collected.

Microbiology events were recorded and the following laboratory results were also collected: lactate value at admission of ICU,the maximum level of blood glucose level, the minimum level of glucose level.

\section{Comorbidities}

We recorded the chronic comorbidities of our study cohort. The MIMICIII database contains over 15,693 different diagnoses classified by ICD 9 and ICD 10 codes. For describing chronic diseases more concisely, we used Elixhauser's comorbidity classification [14] according to an algorithm provided by the authors of the MIMICIII database [15]. Chronic diseases can effectively be reflected by the Elixhauser comorbidity classification, and they have been validated for both ICD 9 and ICD 10 codes $[16,17]$.

\section{Primary and secondary outcomes}

The primary outcome was hospital mortality rate, and the secondary outcomes included number of patients who received MV or RRT during their hospital stay, number of patients received norepinephrine use, length of ICU stay and length of hospital stay, cardiac arrthymias. Cardiac arrhythmias data was collected through discharge diagnosis according to the ICD 9 and ICD 10 codes.

\section{Statistical analysis}

The details of the data screening strategies used are shown in the Additional file 1. Other source codes for our analyses, which were provided by the authors of the MIMICIII database, can be found at GitHub [16, 18]. Categorical variables including demographic data, admission information, and interventions were shown as frequencies, and continuous variables including vital signs and laboratory parameters were presented as mean \pm standard deviation (SD) or median with interquartile range $(25,75 \%)$. We used the analysis of variance or non-parametric tests to analyze continuous variables 
as appropriate. Categorical variables were analyzed using Fisher's exact test or Pearson's chi-square test. We used odds ratios (ORs) and 95\% confidence intervals (CIs) for outcome analysis between groups.

\section{Propensity score matched analysis}

For the primary outcome of hospital mortality, in order to ensure the robustness of our results, we used the propensity score matched (PSM) method to adjust and balance the influence of confounding factors between groups. The variables included in the PSM analysis for matching were as following: age, gender, BMI, MAP, SOFA score, SAPS-I score, SAPS-II score, ECI, sepsis type, admission type, congestive heart failure, coagulopathy disease, lactate level and Glucose-max, norepinephrine use before dobutamine administration, urine volume in the first day of ICU admission. Demographic data and admission information were balanced. Each dobutamine group patient was matched with no dobutamine patients at a proportion of 1:3 with the closest propensity score. The matching caliper was 0.2. PSM analysis required no missing datapoint, so we excluded recorded with data missing. As the data of lactate values are missing a moderate amount, and excluding any patient with a single missing lactate value datapoint will lead to a significant selection bias. Hence, multiple imputation strategies are used for missing lactate values to overcome this deficit in the PSM analysis.

\section{Subgroup analysis}

We also performed subgroup analyses to further investigate whether the primary outcome was different among subgroups. According to the previous studies [19-21] that revealed the risk factors of mortality in sepsis patients and reported the mortality rates might be different among these subgroups, we included the following subgroups: age ( $<60$ years; $\geq 60$ years), BMI ( $\geq 28 \mathrm{~kg} /$ $\left.\mathrm{m}^{2} ;<28 \mathrm{~kg} / \mathrm{m}^{2}\right)$ and gender.

We used PostgreSQL 10.0 software (University of California, Berkeley, California, USA) and Navicat premium 12.0 software (premiumSoft Cybertech Ltd, Kowloon, Hong Kong, China) for database management and data retrieval and screening; R software (version 2.15.x, GNU project) and SPSS 22.0 software (IBM Corp., Armonk, NY, USA) were used for statistical analysis. A two sided $\mathrm{P}<0.05$ was statistical significance.

\section{Results}

Initially, 38,605 patients in the MIMIC III database were screened for eligibility, and 8906 records were included. After removing duplicate records or readmissions to the ICU, 4680 sepsis patients were left. We further screened the demographic data and admission information, and
2394 patients were removed due to information insufficient. Ultimately, 2286 patients were included in this study (Fig. 1). And according to the usage of dobutamine or not, patients were divided into the dobutamine group or the non-dobutamine group. In total, there were 121 patients in the dobutamine group and 2165 patients in the non-dobutamine group.

The clinical characteristics and laboratory results of the included patients are shown in Table 1. Compared to patients in non-dobutamine group, patients in dobutamine group had a significant lower MAP $(46.44 \pm 15.51 \mathrm{mmHg}$ vs. $51.63 \pm 14.01 \mathrm{mmHg}, \mathrm{p}<0.001)$, higher HR $(118.60 \pm 23.46$ beats $/$ min vs.112.71 \pm 22.97 beats/min, $\mathrm{p}=0.006)$, higher $\mathrm{RR}(31.78 \pm 8.00$ breaths/ min vs.30.23 \pm 7.11 breaths $/ \mathrm{min}, \mathrm{p}=0.020$ ), higher SOFA score $(9.91 \pm 3.73$ vs. $7.22 \pm 4.00, \quad \mathrm{p}<0.001)$, higher SOFA-cardiovascular score $(3.23 \pm 1.20$ vs. $2.26 \pm 1.45, \mathrm{p}<0.001)$, SAPS-I score $(24.86 \pm 5.64$ vs. $21.46 \pm 5.68, \mathrm{p}=0.000)$ and SAPS-II score $(56.59 \pm 14.83$ vs. $46.76 \pm 16.66, \mathrm{p}<0.001)$. More patients in dobutamine group received mechanical ventilation at the first day of ICU admission than those in non-dobutamine group (95/121 patients vs. $1128 / 2165$ patients, $\mathrm{p}=0.000)$. Furthermore, more patients in dobutamine group received norepinephrine $(90.9 \%$ vs. $58.6 \%, \mathrm{p}=0.000)$ and the norepinephrine doses were higher in dobutamine group than those in non-dobutamine group $(79.89 \pm 96.44 \mathrm{mg}$ vs. $58.30 \pm 82.56 \mathrm{mg}, \mathrm{p}<0.001)$. The volumes of fluid administered were similar between both groups in the first day of ICU admission. However, the urine volumes in the first day were less in dobutamine group $(1083.63 \pm 1005.80 \mathrm{vs}$. $1565.61 \pm 1333.18 \mathrm{ml}, \mathrm{p}<0.001$ ) (Table 1).

The culture positive rates of microbiology samples were similar between both groups (1719/2165 patients vs. $99 / 121$ patients, $\mathrm{p}=0.521$ ) (Table 1 ). With regards to comorbidities, more patients in dobutamine group had congestive heart failure (75/121 patients vs. $780 / 2165$ patients, $\mathrm{p}<0.001)$ and coagulopathy disease $(53 / 121$ patients vs. $614 / 2165$ patients, $\mathrm{p}<0.001)$ than those in non-dobutamine group (Table 1 ).

\section{Primary outcome and PSM analysis}

For the primary outcome of hospital mortality, 72 of 121 patients $(59.5 \%)$ in the dobutamine group and 754 of 2165 patients $(34.8 \%)$ in the non-dobutamine group died in the hospital, which resulted in a significant betweengroup difference (OR 1.56, 95\% CI 1.01-2.40; $\mathrm{P}=0.000$ ) (Table 2).

For the secondary outcomes, more patients in dobutamine group received MV use, RRT use, norepinephrine use than those in non-dobutamine group (Table 2). Compared with patients in non-dobutamine 


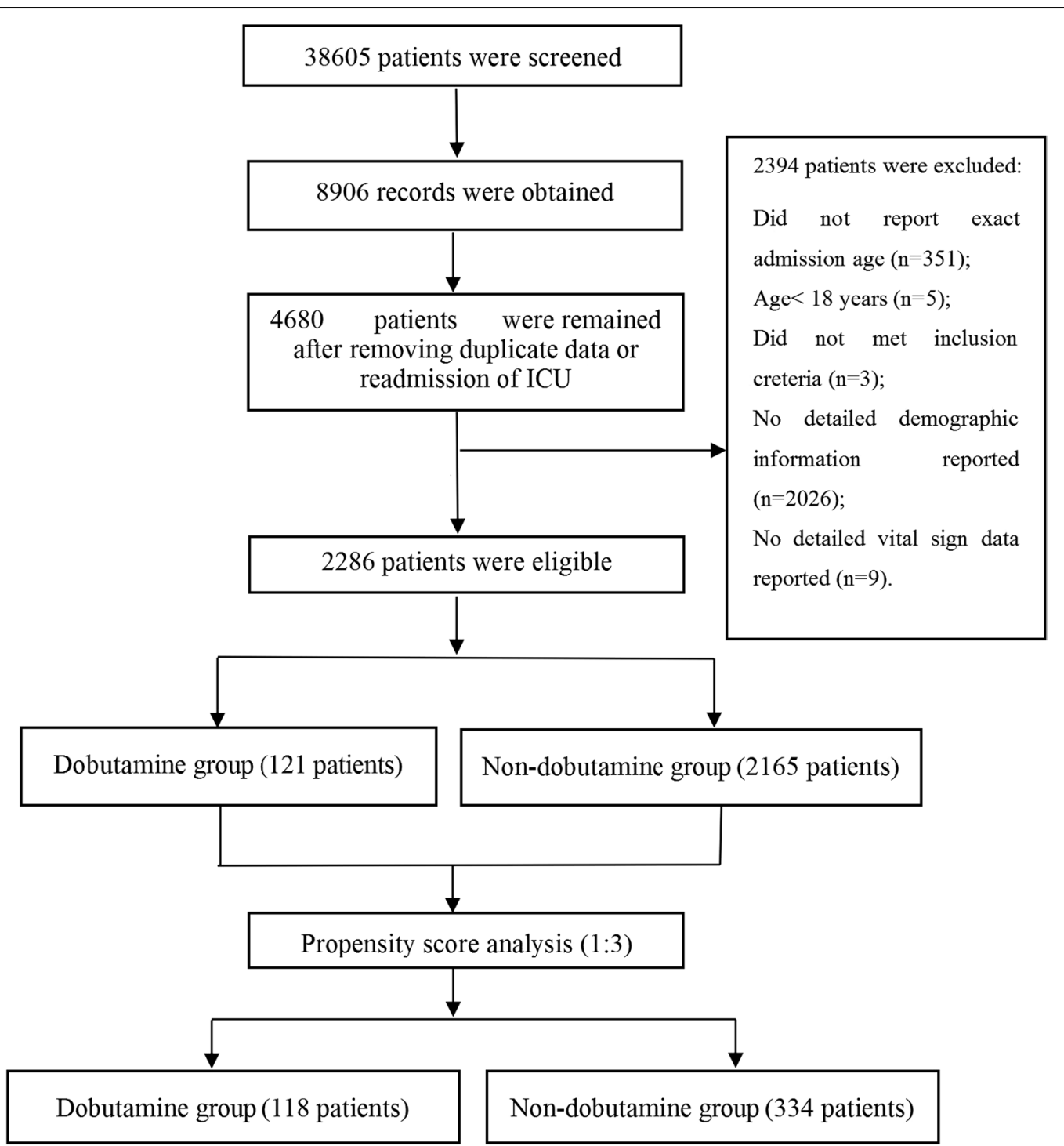

Fig. 1 Study screening and selection process. ICU intensive care unit

group, the duration of ICU stay was longer for patients in dobutamine group (Table 2).

For adverse events, more cardiac arrhythmias occured in dobutamine group than those in non-dobutamine group (Table 2).

In order to test the robustness of the primary and secondary outcomes, a PSM analysis (1:3) was performed. The baseline patients data included in the PSM analysis were shown in Table 3. The PSM analysis showed that results were consistent with hospital mortality and MV use (Table 2).

\section{Subgroup analyses for primary outcomes}

Subgroup analyses with regards to hospital mortality according to gender, age ( $\geq 60$ years, $<60$ years), BMI $\left(\geq 28 \mathrm{~kg} / \mathrm{m}^{2},<28 \mathrm{~kg} / \mathrm{m}^{2}\right)$ showed that there were no significant interactions between subgroups (Fig. 2).

\section{Discussion}

In this retrospective study, a total of 2286 patients with sepsis were included. Compared with patients in nondobutamine group, patients in dobutamine group had a lower MAP, higher HR, higher RR, higher severity of 
Table 1 Baseline demographic data and clinical characteristics of patients included in the study

\begin{tabular}{|c|c|c|c|c|}
\hline \multirow[t]{2}{*}{ Covariate } & \multicolumn{3}{|c|}{ Original patients (before matching) } & \multirow{2}{*}{$\begin{array}{l}\text { Missing } \\
\text { data (\%) }\end{array}$} \\
\hline & $\begin{array}{l}\text { Non-dobutamine group } \\
(\mathrm{N}=2165)\end{array}$ & Dobutamine group $(\mathrm{N}=121)$ & $P$ value & \\
\hline Age (years) & $66.63 \pm 17.06$ & $68.57 \pm 16.44$ & 0.222 & 0.0 \\
\hline Gender (Male), n (\%) & $1208(55.8 \%)$ & $74(61.2 \%)$ & 0.248 & 0.0 \\
\hline Height $(\mathrm{cm})$ & $168.76 \pm 11.00$ & $168.61 \pm 9.68$ & 0.883 & 0.0 \\
\hline Weight (kg) & $82.65 \pm 27.00$ & $83.26 \pm 21.95$ & 0.808 & 0.0 \\
\hline BMI (kg/m [2]) & $29.00 \pm 9.00$ & $29.13 \pm 6.72$ & 0.871 & 0.0 \\
\hline Temperature $\left({ }^{\circ} \mathrm{C}\right)$ & $37.71 \pm 1.04$ & $37.87 \pm 1.11$ & 0.108 & 0.4 \\
\hline MAP $(\mathrm{mmHg})$ & $51.63 \pm 14.01$ & $46.44 \pm 15.51$ & 0.000 & 0.0 \\
\hline HR (beats $\min ^{-1}$ ) & $112.71 \pm 22.97$ & $118.60 \pm 23.46$ & 0.006 & 0.0 \\
\hline $\operatorname{RR}\left(\min ^{-1}\right)$ & $30.23 \pm 7.11$ & $31.78 \pm 8.00$ & 0.020 & 0.0 \\
\hline SOFA score & $7.22 \pm 4.00$ & $9.91 \pm 3.73$ & 0.000 & 0.0 \\
\hline SOFA-cardiovascular score & $2.26 \pm 1.45$ & $3.23 \pm 1.20$ & 0.000 & 0.0 \\
\hline SAPS-I score & $21.46 \pm 5.68$ & $24.86 \pm 5.64$ & 0.000 & 0.0 \\
\hline SAPS-II score & $46.76 \pm 16.66$ & $56.59 \pm 14.83$ & 0.000 & 0.0 \\
\hline $\mathrm{ECl}$ & $10.36 \pm 7.91$ & $11.06 \pm 7.92$ & 0.342 & 0.0 \\
\hline Norepinephrine dose (mg) & $58.30 \pm 82.56$ & $79.89 \pm 96.44$ & 0.004 & 0.0 \\
\hline Norepinephrine use & $1269(58.6 \%)$ & $110(90.9 \%)$ & 0.000 & 0.0 \\
\hline Urine volume (ml, 1st day) & $1565.61 \pm 1333.18$ & $1083.63 \pm 1005.80$ & 0.000 & 1.0 \\
\hline Input volume (ml, 1st day) & $8209.26 \pm 6142.67$ & $9471.47 \pm 7166.17$ & 0.069 & 4.3 \\
\hline \multicolumn{5}{|l|}{ Microbiology, n (\%) } \\
\hline Positive (\%) & $1719(79.4 \%)$ & 99 (81.8\%) & 0.521 & 0.0 \\
\hline Negative & $446(20.6 \%)$ & $22(18.2 \%)$ & & \\
\hline \multicolumn{5}{|l|}{ Interventions, n (\%) } \\
\hline Renal replacement use (1st day) & $170(7.9 \%)$ & $15(12.4 \%)$ & 0.074 & 0.0 \\
\hline Mechanical ventilation use (1st day) & $1128(52.1 \%)$ & $95(78.5 \%)$ & 0.000 & 0.0 \\
\hline \multicolumn{5}{|l|}{ Comorbidities, n (\%) } \\
\hline Congestive heart failure & $780(36.0 \%)$ & 75 (62.0\%) & 0.000 & 0.0 \\
\hline Pulmonary circulation disease & $168(7.8 \%)$ & $10(8.3 \%)$ & 0.840 & 0.0 \\
\hline Renal failure & $469(21.7 \%)$ & $23(19.0 \%)$ & 0.489 & 0.0 \\
\hline Diabetes & $676(31.2 \%)$ & $44(36.4 \%)$ & 0.236 & 0.0 \\
\hline COPD & $496(22.9 \%)$ & $33(27.3 \%)$ & 0.268 & 0.0 \\
\hline Coagulopathy & $614(28.4 \%)$ & $53(43.8 \%)$ & 0.000 & 0.0 \\
\hline Fluid electrolyte disorder & 1204 (55.6\%) & $66(54.5 \%)$ & 0.818 & 0.0 \\
\hline \multicolumn{5}{|l|}{ Admission type, n (\%) } \\
\hline Emergency & $2106(97.3 \%)$ & $113(93.4 \%)$ & 0.014 & 0.0 \\
\hline Elective & $59(2.7 \%)$ & $8(6.6 \%)$ & & 0.0 \\
\hline \multicolumn{5}{|l|}{ Sepsis type, n (\%) } \\
\hline Sepsis & $451(20.8 \%)$ & $7(5.8 \%)$ & 0.000 & 0.0 \\
\hline Severe sepsis & $896(41.4 \%)$ & $50(41.3 \%)$ & & 0.0 \\
\hline Septic shock & $818(37.8 \%)$ & $64(52.9 \%)$ & & 0.0 \\
\hline \multicolumn{5}{|l|}{ Laboratory Tests } \\
\hline Lactate (mmol/L) & $2.59 \pm 2.19$ & $3.50 \pm 2.86$ & 0.001 & 48.4 \\
\hline Glucose-min & $106.90 \pm 38.77$ & $108.06 \pm 48.17$ & 0.753 & 0.5 \\
\hline Glucose-max & $190.70 \pm 94.38$ & $227.50 \pm 93.32$ & 0.000 & 0.5 \\
\hline
\end{tabular}

Data are mean $\pm S D$, median (interquartile) or $\mathrm{n}(\%)$. SOFA Sequential Organ Failure Assessment, ranging from 0 to 24 , with higher scores indicating a greater degree of organ failure; $\mathrm{ECl}$ Elixhauser comorbidity index, and we used the modified vanWalraven Elixhauser comorbidity score in our study, which consists of 30 comorbidity diseases, range from - 19 to 89 points, with higher scores indicating a greater risk of hospital mortality, MAP mean arterial pressure; HR: heart rate, RR respiratory rate, SAPS-I simplified acute physiologic score-I, SAPS-II simplified acute physiologic score-II, ICU intensive care unit, COPD chronic obstructive pulmonary disease. The definitions and diagnositic criteria for sepsis, severe sepsis and septic shock were made according to the Surviving Sepsis Campaign Guidelines [1, 13, 14] 
Table 2 Comparison of primary and secondary outcomes

\begin{tabular}{|c|c|c|c|c|c|c|c|c|}
\hline \multirow[t]{2}{*}{ Outcomes n (\%) } & \multicolumn{4}{|c|}{ Original patients (before matching) } & \multicolumn{4}{|c|}{ PSM adjusted patients (after matching) } \\
\hline & $\begin{array}{l}\text { Non-dobutamine } \\
\text { group }(N=2165)\end{array}$ & $\begin{array}{l}\text { Dobutamine } \\
\text { group } \\
(\mathrm{N}=121)\end{array}$ & OR $(95 \% \mathrm{Cl})$ & $P$ value & $\begin{array}{l}\text { Non- } \\
\text { dobutamine } \\
\text { group }(\mathrm{N}=334)\end{array}$ & $\begin{array}{l}\text { Dobutamine } \\
\text { group } \\
(\mathrm{N}=118)\end{array}$ & OR $(95 \% \mathrm{CI})$ & $P$ value \\
\hline Hospital mortality & $754(34.8 \%)$ & $72(59.5 \%)$ & $2.75(1.89-4.00)$ & 0.000 & $165(49.4 \%)$ & $71(60.2 \%)$ & $1.55(1.01-2.37)$ & 0.044 \\
\hline MV use & $1312(60.6 \%)$ & $108(89.3 \%)$ & $5.40(3.02-9.66)$ & 0.000 & $268(80.2 \%)$ & $105(89.0 \%)$ & $1.99(1.05-3.76)$ & 0.032 \\
\hline RRT use & $208(9.6 \%)$ & $28(23.1 \%)$ & $2.83(1.81-4.43)$ & 0.000 & $56(16.8 \%)$ & $27(22.9 \%)$ & $1.47(0.88-2.47)$ & 0.140 \\
\hline $\begin{array}{l}\text { Norepinephrine } \\
\text { free days }\end{array}$ & $13.01 \pm 13.74$ & $11.47 \pm 11.10$ & $\mathrm{Na}$ & 0.255 & $12.68 \pm 13.54$ & $11.34 . \pm 11.20$ & $\mathrm{Na}$ & 0.360 \\
\hline $\begin{array}{l}\text { Cardiac arrhyth- } \\
\text { mias }\end{array}$ & $912(42.1 \%)$ & $68(56.2 \%)$ & $1.76(1.22-2.55)$ & 0.002 & $171(51.2 \%)$ & $67(56.8 \%)$ & $1.25(0.82-1.91)$ & 0.297 \\
\hline $\begin{array}{l}\text { Duration of ICU } \\
\text { stay (days) }\end{array}$ & $8.08 \pm 9.97$ & $11.77 \pm 11.09$ & $\mathrm{Na}$ & 0.000 & $10.20 \pm 10.43$ & $11.70 \pm 11.18$ & $\mathrm{Na}$ & 0.189 \\
\hline $\begin{array}{l}\text { Duration of hospi- } \\
\text { tal stay (days) }\end{array}$ & $14.98 \pm 15.15$ & $14.98 \pm 12.22$ & $\mathrm{Na}$ & 0.997 & $15.54 \pm 14.22$ & $14.79 \pm 12.29$ & $\mathrm{Na}$ & 0.611 \\
\hline
\end{tabular}

$M V$ mechanical ventilation, $R R T$ renal replacement therapy

illness scores. Furthermore, more patients in dobutamine group received mechanical ventilation at the first day of ICU admission than those in non-dobutamine group. Despite these factors showed that dobutamine group patients were sicker, we found a signifcantly higher hospital mortality among patients who received dobutamine use, and the result was consistant after adjustment for confoundings by PSM analysis. Furthermore, more patients in dobutamine group received MV use, RRT use, had longer durations of ICU stay and more cardiac arrhymias than those in non-dobutamine group.

Dobutamine as an catecholamine working through the $\beta 1$-adrenoceptor- $G \alpha_{s}$ protein- adenylate cyclase- cAMP pathway, is recommended in septic patients with myocardial dysfunction [1]. However, the data supporting the use of dobutamine are mainly physiologic. So far, no randomized controlled trials have performed to evaluate whether there are significant differences between dobutamine and placebo on clinical outcomes in septic patients [1]. Further researches regarding the effects of dobutamine on patient centered outcomes such as mortality are warranted [22].

Our studies showed a signifcantly higher hospital mortality among dobutamine group than non dobutamine group, and the result was consistant after adjustment for confoundings by PSM analysis. Our findings was similar with a previous study by Wilkman and colleagues, in which patients received inotrope use had a higher 90 -day mortality $(42.5 \%$ vs. $23.9 \%, \mathrm{P}<0.001)$ and hospital mortality $(18.5 \%$ vs. $33.8 \%, \mathrm{P}<0.001)$ than those without [23]. The hospital mortality was higher in our study, which might be due to patients in our study were older than those in Wilkman's study $(66.63 \pm 17.06$ years in non-dobutamine group and $68.57 \pm 16.44$ years in dobutamine group in our study, $53.3 \pm 13.9$ years in no inotropes group and $56.4 \pm 14.6$ years in inotropes group in Wilkman's study). In addition, in our study, the overall mortality rate of sepsis patients was $36.1 \%$ (95\% CI $34.2 \%-38.1 \%$ ), which was similar with the rates reported in other large-scale studies $[24,25]$, demonstrating the high reliability of our study.

Furthermore, this study showed that patients in dobutamine group received more MV and RRT therapy, had longer durations of ICU stay and more cardiac arrhymas than those in non-dobutamine group. These results demonstrated that benefits of dobutamine use may have been overemphasized.

One explanation for our findings might be that effect of dobutamine as a positive inotrope is impaired in sepsis. Mari and colleagues found that the cardial responsiveness to dobutamine inotropic effect was significantly diminished in septic mice model compared with shamoperated controls [26]. They further found that this was related to upregulated phosphodiesterase 4D leading to plasma cAMP breakdown. Understanding the effect of phosphodiesterase 4D in regulating cardiac responsiveness to dobutamine may provide the potential of a PDE4D targeted therapy for sepsis patients with low cardiac output requiring inotropic support in future. Another explanation might be that the use of dobutamine was associated with adverse effect. A previous study showed that dobutamine also may dobutamine may promote inflammatory response by increasing circulating TNF-a levels in patients with septic shock [27]. The use of catecholamines could decrease metabolic efficiency and increase bacterial growth [6]. Furthermore, the use of dobutamine to increase oxygen delivery to a supernormal target did not improve mortality in critically ill 
Table 3 Baseline demographic data and clinical characteristics of patients included in the PSM analysis

\begin{tabular}{|c|c|c|c|}
\hline \multirow[t]{2}{*}{ Covariate } & \multicolumn{3}{|l|}{ Original patients (after matching) } \\
\hline & Non-dobutamine group $(\mathrm{N}=334)$ & Dobutamine group $(\mathrm{N}=118)$ & $P$ value \\
\hline Age (years) & $70.93 \pm 19.89$ & $68.48 \pm 16.53$ & 0.231 \\
\hline Gender (Male), n (\%) & $204(61.1 \%)$ & $71(60.2 \%)$ & 0.862 \\
\hline Height(cm) & $168.78 \pm 10.80$ & $168.48 \pm 9.70$ & 0.787 \\
\hline Weight (kg) & $82.08 \pm 26.41$ & $83.06 \pm 22.16$ & 0.719 \\
\hline BMI $\left(\mathrm{kg} / \mathrm{m}^{2}\right)$ & $28.75 \pm 8.59$ & $29.10 \pm 6.80$ & 0.683 \\
\hline Temperature $\left({ }^{\circ} \mathrm{C}\right)$ & $37.63 \pm 1.16$ & $37.88 . \pm 1.12$ & 0.044 \\
\hline MAP $(\mathrm{mmHg})$ & $47.38 \pm 15.37$ & $46.46 \pm 15.69$ & 0.579 \\
\hline HR (beats $\min ^{-1}$ ) & $115.41 \pm 23.62$ & $118.93 \pm 23.28$ & 0.163 \\
\hline $\operatorname{RR}\left(\min ^{-1}\right)$ & $30.69 \pm 7.56$ & $31.75 \pm 8.09$ & 0.199 \\
\hline SOFA score & $9.66 \pm 3.93$ & $9.87 \pm 3.73$ & 0.606 \\
\hline SOFA-cardiovascular score & $3.13 \pm 1.25$ & $3.21 \pm 1.20$ & 0.517 \\
\hline SAPS-I score & $24.50 \pm 5.69$ & $24.83 \pm 5.63$ & 0.590 \\
\hline SAPS-II score & $56.55 \pm 16.63$ & $56.39 \pm 14.87$ & 0.925 \\
\hline $\mathrm{ECl}$ & $11.00 \pm 7.47$ & $11.21 \pm 7.91$ & 0.797 \\
\hline Norepinephrine dose (mg) & $64.97 \pm 90.54$ & $75.78 \pm 89.60$ & 0.289 \\
\hline Norepinephrine use & $305(91.3 \%)$ & $107(90.7 \%)$ & 0.833 \\
\hline Urine volume (ml, 1st day) & $1169.60 \pm 1123.08$ & $1096.28 \pm 1011.60$ & 0.534 \\
\hline Input volume (ml, 1st day) & $9500.05 \pm 7096.23$ & $9488.29 \pm 7257.87$ & 0.988 \\
\hline \multicolumn{4}{|l|}{ Microbiology, n (\%) } \\
\hline Positive (\%) & $275(82.6 \%)$ & $96(81.4 \%)$ & 0.764 \\
\hline Negative & $58(17.4 \%)$ & $22(18.6 \%)$ & \\
\hline \multicolumn{4}{|l|}{ Interventions, n (\%) } \\
\hline Renal replacement use (1st day) & $41(12.3 \%)$ & $14(11.9 \%)$ & 0.907 \\
\hline Mechanical ventilation use (1st day) & $244(73.1 \%)$ & $92(78.0 \%)$ & 0.294 \\
\hline \multicolumn{4}{|l|}{ Comorbidities, n (\%) } \\
\hline Congestive heart failure & $204(61.1 \%)$ & $73(61.9 \%)$ & 0.880 \\
\hline Pulmonary circulation disease & $21(6.3 \%)$ & $10(8.5 \%)$ & 0.419 \\
\hline Renal failure & $77(23.1 \%)$ & $22(18.6 \%)$ & 0.319 \\
\hline Diabetes & $112(33.5 \%)$ & $43(36.4 \%)$ & 0.567 \\
\hline COPD & $81(24.3 \%)$ & $33(28.0 \%)$ & 0.424 \\
\hline Coagulopathy & $137(41.0 \%)$ & $51(43.2 \%)$ & 0.676 \\
\hline Fluid electrolyte disorder & $166(49.7 \%)$ & $66(55.9 \%)$ & 0.244 \\
\hline \multicolumn{4}{|l|}{ Admission type, n (\%) } \\
\hline Emergency & $310(92.8 \%)$ & 110 (93.2\%) & 0.883 \\
\hline Elective & $24(7.2 \%)$ & $8(6.8 \%)$ & \\
\hline \multicolumn{4}{|l|}{ Sepsis type, n (\%) } \\
\hline Sepsis & $18(5.4 \%)$ & $7(5.9 \%)$ & 0.913 \\
\hline Severe sepsis & $143(42.8 \%)$ & $48(40.7 \%)$ & \\
\hline Septic shock & 173 (51.8\%) & $63(53.4 \%)$ & \\
\hline \multicolumn{4}{|l|}{ Laboratory tests } \\
\hline Lactate (mmol/L) & $3.01 \pm 2.56$ & $3.55 \pm 2.49$ & 0.047 \\
\hline Glucose-min & $104.74 \pm 40.59$ & $108.08 \pm 48.52$ & 0.466 \\
\hline Glucose-max & $211.77 \pm 112.57$ & $225.84 \pm 93.33$ & 0.224 \\
\hline
\end{tabular}

Data are mean $\pm S D$, median (interquartile) or $\mathrm{n}(\%)$. SOFA Sequential Organ Failure Assessment, ranging from 0 to 24 , with higher scores indicating a greater degree of organ failure, $\mathrm{ECl}$ Elixhauser comorbidity index, and we used the modified vanWalraven Elixhauser comorbidity score in our study, which consists of 30 comorbidity diseases, range from - 19 to 89 points, with higher scores indicating a greater risk of hospital mortality, $M A P$ mean arterial pressure, $H R$ heart rate, $R R$ respiratory rate, SAPS-I simplified acute physiologic score-I; SAPS-II simplified acute physiologic score-II, ICU intensive care unit, COPD chronic obstructive pulmonary disease. The definitions and diagnositic criteria for sepsis, severe sepsis and septic shock were made according to the Surviving Sepsis Campaign Guidelines [1, 13, 14]. No variable had a missing value 


\begin{tabular}{|c|c|c|c|c|c|c|c|}
\hline Subgroup & $\begin{array}{l}\text { No. of } \\
\text { patients }\end{array}$ & $\begin{array}{l}\text { Non-dobutamine } \\
\text { group } \\
\text { no./total no. (\%) }\end{array}$ & $\begin{array}{l}\text { Dobutamine } \\
\text { group } \\
\text { no./total no. (\%) }\end{array}$ & & $\begin{array}{l}\text { Unadjusted Odds Ratio } \\
\qquad(95 \% \mathrm{CI})\end{array}$ & $P$ value & $\begin{array}{l}\text { Interaction of } \\
\text { subgroup } \\
\text { (P value) }\end{array}$ \\
\hline Overall & 2286 & $754 / 2165(34.8)$ & $72 / 121(59.5)$ & $\longrightarrow$ & $2.75(1.89-4.00)$ & 0.000 & \\
\hline Gender & & & & & & & 0.69 \\
\hline Male & 1282 & $419 / 1208(34.7)$ & $45 / 74(60.8)$ & & $2.92(1.81-4.73)$ & 0.000 & \\
\hline Female & 1004 & $335 / 957(35.0)$ & $27 / 47(57.4)$ & \begin{tabular}{l|l} 
& $-\square-$
\end{tabular} & $2.51(1.39-4.54)$ & 0.002 & \\
\hline BMI $\left(\mathrm{kg} / \mathrm{m}^{2}\right)$ & & & & & & & 0.93 \\
\hline$<28$ & 1251 & $423 / 1189(35.6)$ & $38 / 62(61.3)$ & $\mapsto-\square$ & $2.87(1.70-4.85)$ & 0.000 & \\
\hline$\geq 28$ & 1034 & $331 / 975(33.9)$ & $34 / 59(57.6)$ & $\longmapsto$ & $2.65(1.55-4.51)$ & 0.000 & \\
\hline Age(years) & & & & & & & 0.92 \\
\hline$<60.00$ & 742 & 201/709(28.3) & $16 / 33(48.5)$ & $-\square-$ & $2.38(1.18-4.80)$ & 0.000 & \\
\hline \multirow[t]{2}{*}{$\geq 60.00$} & 1544 & $553 / 1456(38.0)$ & $56 / 88(63.6)$ & $-\mathbf{n}-$ & $2.86(1.83-4.47)$ & 0.013 & \\
\hline & & & & $\begin{array}{llll}00 & 2.00 & 3.00 & 4.0 \\
\end{array}$ & & & \\
\hline
\end{tabular}

Fig. 2 Subgroup analyses with regard to hospital mortality. BMI body mass index

patients $[28,29]$. There are also some evidences that inotropic drugs are associated with worse outcomes in heart failure and cardiac surgery patients [30-32].

Some studies reported the adverse effects caused by inotropes, including myocardial oxygen consumption increase, higher incidence of arrhythmias and myocardial ischemia $[7,8,33,34]$. In a randomized controlled trial, 516 patients with septic shock were randomly assigned to placebo group or levosimendan group; no difference was found in mortality. However, there was more tachyarrhythmias in levosimendan group than placebo group (absolute difference, 2.7\%, 95\% CI 0.1-5.3\%) [35]. Our study also found that more cardiac arrhythmias occured in dobutamine group compared with non-dobutamine group.

The advantages of this study were that we performed a big data, large scale research based on the MIMIC III database, included patients with rigorous criteria, and performed subgroup and propensity score matched analysis to adjust for confoundings between the groups, which increased the reliability of the primary and secondary outcomes.

Our study has several limitations. First, our study was based on the analysis of MIMICIII database. Due to the retrospective nature of this study, there was unavoidable risk of bias. To decrease the influences of selection bias of more critically ill patients tending to receive dobutamine, our study adjusted for the baseline characteristics between non-dobutamine and dobutamine groups by propensity score matched method, and we further studied the primary outcomes through multiple subgroup analyses. Ultimately, the results were still consistent, which demonstrated that our results were reliable. Second, sepsis-induced cardiomyopathy (SIC) was reported in many studies which was characterised with left ventricular diastolic dysfunction and depressed ejection fraction. The incidence rate of SIC was high, approximately $24 \%-40 \%$ [36, 37]. Furthermore, some studies showed that diastolic dysfunction might be aggravated by catecholamines $[38,39]$. Hence, some septic patients with diastolic dysfunction might account for a large proportion of patients who used dobutamine. And these patients might not benefit from dobutamine use. However, this aspects was not assessed in this study. Third, several hemodynamic variables as CVP, pulmonary capillary wedge pressure (PCWP) or cardiac index (CI) were not included in this study due to data insuffecient, which might influence the analysis of possible effects of dobutamine. However, a thorough physiologic and clinical examination variables (heart rate, temperature, respiratory rate, mean blood pressure) were included in our study. Furthermore, this study raised the concerns about dobutamine use in septic patients. Further large scale, randomized controlled studies are warrented to confirm our findings.

\section{Conclusion}

Among patients with sepsis, our study showed that the use of dobutamine was not associated with decreased hospital mortality. The mechanism remains to be explored. Further large scale, randomized controlled studies are warrented to confirm our findings.

\section{Abbreviations}

BMI: Body mass index; SOFA: Sequential Organ Failure Assessment; ECI: Elixhauser comorbidity index; MAP: Mean arterial pressure; HR: Heart rate; RR: Respiratory rate; SAPS-I: Simplified acute physiologic score-l; SAPS-II: Simplified acute physiologic score-ll; ICU: Intensive care unit; COPD: Chronic obstructive pulmonary disease; ORs: Odds ratios; Cl: Confidence intervals; PSM: Propensity score matched analysis. 


\section{Supplementary Information}

The online version contains supplementary material available at https://doi. org/10.1186/s12879-021-06852-8.

Additional file 1: Figure S1. Distribution of propensity scores between treatment group (Dobutamine group) and control group (Non-dobutamine group) before and after match. Figure $\mathbf{S 2}$. The absolute standardized difference in Means before and after match. Figure S3. The density of propensity score between treatment group (Dobutamine group) and control group (Non-dobutamine group) before and after match. Figure S4. The distribution of standardized difference in Means before and after match. Figure $\mathbf{S 5}$. The propensity score of variables between treatment group (Dobutamine group) and control group (Non-dobutamine group) before and after match. Data Secreening Strategy

\section{Acknowledgements}

We acknowledge all staffs who helped us in performing this study and we particularly aknowledege professor Shaoheng Zhang who provided advice in data analysis.

\section{Authors' contributions}

All authors contributed to the study conception and design. YFZ, RZ and HYY performed the research. XLY collected and analyzed the data. YFZ, RZ and JRW wrote the manuscript. All authors read and approved the final manuscript.

\section{Funding}

This study was supported by the Science and Technology Program of Guang zhou Science, Technology, and Innovation Commission (201904010258). The funding body was not involved in the design of the study and collection, analysis, and interpretation of data and in writing the manuscript.

\section{Availability of data and materials}

The Datasets are available from the corresponding author on reasonable request. The details of the data screening strategies used are shown in the supplementary file. Other source codes for our analyses, which were provided by the authors of the MIMICIII database, can be found at GitHub (https:// github.com/MIT-LCP/mimic-code).

\section{Declarations}

\section{Ethics approval and consent to participate}

As the present study was based on the analysis of MIMICIII public database, ethical review and informed consent were granted to waive.

\section{Consent for publication}

Not applicable.

\section{Competing interests}

The authors declare no competing interests.

\section{Author details}

'Department of Intensive Care Unit, Guangzhou Red Cross Hospital, Medical College, Jinan University, Guangdong 510220 Guangzhou, China. ${ }^{2}$ Department of Critical Care Medicine, The First Affiliated Hospital of Jinan University, Guangzhou 510220, Guangdong, China. ${ }^{3}$ Guangzhou Women and Children's Medical Center, No. 9 Jinsui Road, Guangzhou 510220, Guangdong, China.

Received: 4 November 2020 Accepted: 9 November 2021

Published online: 11 November 2021

\section{References}

1. Andrew R, Laura EE, Waleed A, Mitchell ML, Massimo A, Ricard F, et al. Surviving sepsis campaign: international guidelines for management of sepsis and septic shock: 2016. Intensive Care Med. 2017;43(3):304-77.
2. Mouncey PR, Osborn TM, Power GS, Harrison DA, Sadique MZ, Grieve RD, et al. Trial of early, goal-directed resuscitation for septic shock. N Engl J Med. 2015;372(14):1301-11.

3. The ProCESS Investigators. A randomized trial of protocol-based care for early septic shock. N Engl J Med. 2014;370(18):1683-93.

4. Peake SL, Delaney A, Balley M, Bellomo R, Cameron PA, Investigators ARISE, ANZICS Clinical Trial Group, et al. Early goal-directed therapy in the treatment of severe sepsis and septic shock. N Engl J Med. 2014:371(16):1496-506.

5. Andreis DT, Khaliq W, Singer M. Acute haemodynamic effects of dobutamine in experimental sepsis-induced myocardial depression. Intensive Care Med Exp. 2015;3(Suppl 1):A799.

6. Singer M. Catecholamine treatment for shock-equally good or bad? Lancet. 2007;370:636-7.

7. William TL, Aalap N, Brian WH. Dobutamine induced eosinophilic cardiomyopathy. JACC. 2016;67(13):1047.

8. Hajsadeghi S, Rahbar MH, Iranpour A, Salehi A, Asadi O, Jafarian SR. Dobutamine-induced takotsubo cardiomyopathy: a systematic review of the literature and case report. Anatol J Cardiol. 2018;19:412-6.

9. John EM. Pathophysiological insights from dobutamine-induced takotsubo syndrome? Anatol J Cardiol. 2018. https://doi.org/10.14744/Anato IJCardiol.2018.50708.

10. Erik VE, Douglas GA, Matthias E, Stuart JP, Peter CG, Jan PV, for the STROBE Initiative. The strengthening the reporting of observational studies in epidemiology (STROBE) statement: guidelines for reporting observational studies. PLOS Med. 2007;4(10):e296.

11. Johnson AEW, Pollard TJ, Shen L, Lehman L, Feng M, Ghassemi M, Moody B, Szolovits P, Celi LA, Mark RG. MIMIC-III, a freely accessible critical care database. Sci Data. 2016;2016:160035.

12. Goldberger AL, Amaral LAN, Glass L, Hausdorff JM, Ivanov PCh, Mark RG, Mietus JE, Moody GB, Peng C-K, Stanley HE. PhysioBank, physiotoolkit, and physionet: components of a new research resource for complex physiologic signals. Circulation. 2000;101(23):e215-20.

13. Dellinger RP, Levy MM, Rhodes A, et al. Surviving Sepsis Campaign: international guidelines for management of severe sepsis and septic shock, 2012. Intensive Care Med. 2013;39(2):165-228.

14. Singer $M$, Deutschman CS, Seymour CW, Shankar-Hari M, Annane D, Bauer $\mathrm{M}$, et al. The Third International Consensus definitions for sepsis and septic shock (Sepsis-3). JAMA. 2016;315(8):801-10.

15. Van Walraven C, Austin PC, Jennings A, Quan H, Forster AJ. A modification of the Elixhauser comorbidity measures into a point system for hospital death using administrative data. Med Care. 2009:47:626-33.

16. https://github.com/MIT-LCP/mimic-code. Accessed 29 Oct 2019.

17. Quan H, Sundararajan V, Halfon P, Fong A, Burnand B, Luthi JC, et al. Coding algorithms for defining comorbidities in ICD-9-CM and ICD-10 administrative data. Med Care. 2005:43:1130-9.

18. Johnson AE, Stone DJ, Celi LA, Pollard TJ. The MIMIC code repository: enabling reproducibility in critical care research. JAMA. 2018;25:32-9.

19. Fathi M, Markazi-Moghaddam N, Ramezankhani A. A systematic review on risk factors associated with sepsis in patients admitted to intensive care units. Aust Crit Care. 2019:32(2):155-64.

20. Ahiawodzi PD, Kelly K, Massengill A, Thompson DK. Risk factors for sepsis morbidity in a rural hospital population: a case-control study. AM J INFECT CONTROL. 2018;46(9):1041-6.

21. Martin-Loeches I, Guia MC, Vallecoccia MS, Suarez D, Ibarz M, Irazabal $\mathrm{M}$, et al. Risk factors for mortality in elderly and very elderly critically ill patients with sepsis: a prospective, observational, multicenter cohort study. Ann Intensive Care. 2019;9(1):26.

22. Ryota S, Michitaka N. Time to re-think the use of dobutamine in sepsis. $J$ Intensive Care. 2017:5:65.

23. Wilkman E, Kaukonen KM, Pettilä V, Kuitunen A, Varpula M. Association between inotrope treatment and 90-day mortality in patients with septic shock. Acta Anaesthesiol Scand. 2013;57(4):431-42.

24. Dombrovskiy VY, Martin AA, Sunderram J, et al. Rapid increase in hospitalization and mortality rates for severe sepsis in the United States: a trend analysis from 1993 to 2003. Crit Care Med. 2007:35(5):1244-50.

25. Martin GS, Mannino DM, Eaton S, et al. The epidemiology of sepsis in the United States from 1979 through 2000. N Engl J Med. 2003;348(16):1546-54.

26. Mari S, Tokiko S, Kengo T, Shigeyuki Y, Sailesh P, Kohshi H, et al. Diminished responsiveness to dobutamine as an inotrope in mice with cecal 
ligation and puncture-induced sepsis: attribution to phosphodiesterase 4 upregulation. Am J Physiol Heart Circ Physiol. 2017;312:H1224-7.

27. Hartemink KJ, Groeneveld AB. Vasopressors and inotropes in the treatment of human septic shock: effect on innate immunity? Inflammation. 2012;35:206-13

28. Gattinoni L, Brazzi L, Pelosi P, Latini R, Tognoni G, Pesenti A, Fumagalli R. A trial of goal-oriented hemodynamic therapy in critically ill patients. SvO2 Collaborative Group. N Engl J Med. 1995;333:1025-32.

29. Hayes MA, Timmins AC, Yau EH, Palazzo M, Hinds CJ, Watson D. Elevation of systemic oxygen delivery in the treatment of critically ill patients. N Engl J Med. 1994;330:1717-22.

30. Shahin J, Devarennes B, Tse CW, Amarica DA, Dial S. The relationship between inotrope exposure, six-hour postoperative physiological variables, hospital mortality and renal dysfunction in patients undergoing cardiac surgery. Crit Care. 2011;15:R162.

31. Abraham WT, Adams KF, Fonarow GC, Costanzo MR, Berkowitz RL, et al. In-hospital mortality in patients with acute decompensated heart failure requiring intravenous vasoactive medications: an analysis from the Acute Decompensated Heart Failure National Registry (ADHERE). J Am Coll Cardiol. 2005:46:57-64.

32. Elkayam U, Tasissa G, Binanay C, Stevenson LW, Gheorghiade M, Warnica JW, et al. Use and impact of inotropes and vasodilator therapy in hospitalized patients with severe heart failure. Am Heart J. 2007;153:98-104.

33. Kumar A, Schupp E, Bunnell E, Ali A, Milcarek B, Parrillo JE. Cardiovascular response to dobutamine stress predicts outcome in severe sepsis and septic shock. Crit Care. 2008;12:R35.
34. Jellema WT, Groeneveld AB, Wesseling KH, Thijs LG, Westerhof N, van Lieshout JJ. Heterogeneity and prediction of hemodynamic responses to dobutamine in patients with septic shock. Crit Care Med. 2006;34:2392-8.

35. Gordon AC, Perkins GD, Singer M, et al. Levosimendan for the prevention of acute organ dysfunction in sepsis. N Engl J Med. 2016;375:1638-48.

36. Narváeza I, Canabal A, Martín C, Sánchez M, Moron A, Alcalá J, et al. Incidence and evolution of sepsis-induced cardiomyopathy in a cohort of patients with sepsis and septic shock. Med Intensiva. 2018;42(5):283-91.

37. Ryota S, Akira K, Tadaaki T, Michitaka N, Sarah KL. Prevalence and risk factors of sepsis-induced cardiomyopathy: a retrospective cohort study. Medicine. 2016. https://doi.org/10.1097/MD.00000000000005031.

38. Budhwani N, Bonaparte KL, Cuyjet AB, Saric M. Severe reversible left ventricular systolic and diastolic dysfunction due to accidental iatrogenic epinephrine overdose. Rev Cardiovasc Med. 2004;5:130-3.

39. Rider OJ, Francis JM, Ali MK, Holloway C, Pegg T, Robson MD, et al. Effects of catecholamine stress on diastolic function and myocardial energetics in obesity. Circulation. 2012;125:1511-9.

\section{Publisher's Note}

Springer Nature remains neutral with regard to jurisdictional claims in published maps and institutional affiliations.
Ready to submit your research? Choose BMC and benefit from:

- fast, convenient online submission

- thorough peer review by experienced researchers in your field

- rapid publication on acceptance

- support for research data, including large and complex data types

- gold Open Access which fosters wider collaboration and increased citations

- maximum visibility for your research: over $100 \mathrm{M}$ website views per year

At BMC, research is always in progress.

Learn more biomedcentral.com/submissions 